\title{
IMPORTANCE HAS BEEN CONSIDERED IN SATISFACTION EVALUATION: AN EXPERIMENTAL EXAMINATION OF LOCKE'S RANGE-OF-AFFECT HYPOTHESIS*
}

(Accepted 23 March 2006)

\begin{abstract}
Importance weighting is a common practice in quality of life (QOL) measurement research. Based on the widespread idea that important domains should make a greater contribution to individuals' QOL total score, the weighting procedure of multiplying item satisfaction by an item's importance has been adopted in many QOL instruments. Locke's [1969, Organizational Behavior and Human Performance 4, 309-336; 1976, Handbook of Industrial and Organizational Psychology. Rand McNally, Chicago, pp. 1297-1343] range-of-affect hypothesis indicated that a satisfaction evaluation is determined by the have-want discrepancy, importance, and the interaction of the have-want discrepancy and importance, implying that a satisfaction evaluation incorporates the judgment of importance and weighting the satisfaction score with the importance score is unnecessary. The purpose of the current study was to address the issue of importance weighting by examining Locke's range-of-affect hypothesis in the context of QOL research. A within-subject experiment was conducted to see if, given a varying amount of discrepancy, participants would reveal whether or not satisfaction/dissatisfaction is related to the dimension of importance placed on the object. Forty undergraduate students at National Taiwan University participated in the current study. Results revealed that the association between have-want discrepancy and object satisfaction is stronger on the high important dimension than the less important dimension. Generally, the results were consistent with Locke's range-of-affect hypothesis, revealing that a satisfaction evaluation has incorporated the judgment of item importance, suggesting that the procedure of importance weighting is unnecessary.
\end{abstract}

KEY WORDS: importance, quality of life, satisfaction, weighting

\section{INTRODUCTION}

"Importance weighting" was a common practice in Quality of life (QOL) research. The basic idea was that items for a specific life areas contained in a QOL measurement would have different importances for different individuals; therefore, in recording participants' perception, feeling, or evaluation on these items, the information of item importance should be incorporated

* This paper was a part of the first author's Master's Thesis. 
in the scoring procedure and reflected in the final score. Accordingly, "Importance weighting" was proposed to serve this purpose and the common procedure has been to weigh the satisfaction score by the importance score for each item. Frisch et al. (1992) described this idea as follows:

The inventory's (the Quality of Life Inventory) scoring scheme reflects the assumption that a person's overall life satisfaction is a composite of the satisfactions in particular areas of life weighted by their relative importance to the individual. Thus, the product of the satisfaction and importance ratings for each area of life are computed (p. 93).

This idea has become so common that many QOL instruments, such as the Comprehensive Quality Of Life Scale (ComQol) (Cummins, 1997), the Quality of life Index (QOL Index) (Ferrans and Powers, 1985), the Quality of Life Inventory (QOL Inventory) (Frisch, 1992), the Quality of Life Profile-Adolescent version (QOLPAV) (Raphael et al., 1996), and the Flanagan Quality of Life Scale (QOLS) as reviewed in Dijkers (2003), to name a few, adopted this weighting procedure in their scoring system without examining the necessity and appropriateness of this weighting procedure. The basic assumption behind the importance-weighting procedure was that people evaluate satisfactions on different domains with the same psychological criterion and importance judgment was distinct and independent from satisfaction evaluation, thus, in order to capture the nature of different life domains having different impacts on personal QOL, satisfaction evaluation and importance judgment should be incorporated to gain a single-weighed QOL score.

However, the assumption behind the importance-weighting procedure was not tenable. In fact, literature in psychology has indicated that importance judgment has been considered in satisfaction evaluation. That is, importance judgment and satisfaction evaluation were not two independent processes. For example, Locke $(1969,1976)$ indicated a meaning of importance rating in the context of satisfaction evaluation and discussed the appropriateness of importance weighting on satisfaction scores. He proposed the range-of-affect hypothesis to suggest that responses of satisfaction evaluation reflect a dual value judgment: (a) the discrepancy between what the individual wants and what he/she perceives himself/herself as getting, and (b) the importance to the individual of what is wanted. He further proposed that the level of satisfaction was influenced by the interaction of the have-want discrepancy with importance. Specifically, Locke $(1969,1976)$ claimed that given the amount of discrepancy, an item with high personal importance could produce a wide affective reaction ranging from great satisfaction to great dissatisfaction, and an item with low personal 
importance, on the other hand, could only produce a restricted affective reaction to the neutral point of the satisfaction-dissatisfaction dimension. In brief, his point was, given the amount of discrepancy, the range of the satisfaction rating on an item is determined by the item's importance. Therefore, according to Locke, the range-of-affect hypothesis asserted that item satisfaction has incorporated the information of item importance, which rendered weighting item satisfaction with item importance redundant.

The range-of-affect hypothesis has been supported by empirical studies on job satisfaction, in which the results showed that when a direct cognitive comparison between have and want was made, the strength of item satisfaction was determined by item importance (Mobley and Locke, 1970; Rice et al., 1991; Rice et al., 1991; McFarlin and Rice, 1992; McFarlin et al., 1995). Additionally, in QOL research, Wu and Yao's (in press a) study showed that the range-of-affect hypothesis was also supported in the context of life satisfaction. However, except for Mobley and Locke's (1970) study, research examining the range-of-affect hypothesis has been conducted by between-subject designs rather than within-subject design (e.g., Mobley and Locke, 1970; Rice et al., 1991a; Rice et al., 1991b; McFarlin and Rice, 1992; McFarlin et al., 1995; Wu and Yao, in press a). Most of these studies used regression analysis to show that item have-want discrepancy and item importance have a significant interaction effect in predicting item satisfaction. In this context, the range-of-affect hypothesis stated that the relationship between item have-want discrepancy and item satisfaction was expected to be stronger among people attaching high importance to the said item than to people attaching low importance to that item.

However, a within-subject design was more desirable than a betweensubject design in discussing the issue of importance weighting on satisfaction scores because an individual's QOL score was usually computed from his/ her scores of various life areas. Specifically, a common procedure of importance weighting on satisfaction scores was to multiply importance and satisfaction scores for an item for an individual. If there were 10 life areas, then an individual's QOL score was computed by multiplying importance and satisfaction scores of the 10 life areas and averaging or summing these 10 weighted scores. In this within-subject situation, it is worth investigating if the strength of satisfaction/dissatisfaction of a life area was stronger on more important areas than on less important areas for an individual, when the have-want discrepancy of each area was controlled. If Locke's hypothesis was supported in a within-subject design study, it could reveal that the perception of importance has been considered when an individual makes a satisfaction evaluation of a life area, and then, it could be suggested that 
weighting the item satisfaction score with item importance is unnecessary for an individual. Thus, the main purpose of this study was to examine Locke's range-of-affect hypothesis by conducting within-subject experiments to see if the relationship between item have-want discrepancy and item satisfaction is stronger on the higher-importance item than the lowerimportance one.

In fact, Locke (1969) and Mobley and Locke (1970) have examined the range-of-affect hypothesis by conducting within-subject experiments. Locke (1969) manipulated the level of importance of a reaction time task with payment (high importance) and non-payment (low importance) for each successful trial. As predicted, participants' satisfaction or dissatisfaction of their performance was more extreme in a payment condition than a nonpayment condition. However, in the manipulation check, only 7 out of 21 participants reported that the payment condition was more important than the non-payment condition. Thus, the study was not able to confidently attribute the findings to the level of importance, because the importance manipulation was not successful.

In addition, Mobley and Locke (1970) conducted three within-subject experiments (Study 2 to Study 4) in their research. In Study 2 of Mobley and Locke's (1970) research, participants were asked to select the most important and least important items out of 10 items. And then, with these two items, participants were asked to indicate (a) the alternative which best described what his/her job is or was like (real status) on a 7-point verbally anchored scale, (b) the alternative which best described what the job should be or should have been like (want status) on the same 7-point verbally anchored scale, and (c.) the degree of satisfaction with each of the seven alternatives specified in the scale, that is, the anticipated satisfaction (measured on a 21-point bipolar scale) if they engage in each alternative. From these ratings, item discrepancies of the two items were indicated by the gap between real alternative and want alternative, and were divided into five categories $(0,-1,-2,-3$, and -4 ; other discrepancy levels were ignored). In addition, the satisfaction score for the real alternative represented actual satisfaction. Further, for each discrepancy level, the mean of item satisfaction score across participants was calculated separately for the most and least important items. In this procedure, only participants with at least one of the five discrepancy categories for both items could be included. Therefore, of 102 participants, data of only 34 was used. Although the result of this study supported the range-of-affect hypothesis, revealing that the relation between item discrepancy and item satisfaction was stronger for the most important than for least important item, the method of analysis 
assumed that the same discrepancy unit obtained from different items has the same meaning. In addition, the analysis method lost too much information. Therefore, this is not an ideal experiment to examine the hypothesis.

In Study 3 of Mobley and Locke's (1970) research, they manipulated the high-low importance by two forms of a question, in which intrinsic interest was used to manipulate the level of importance, assuming that question content involving intrinsic interest would be more important. Participants were asked to indicate (a) the alternative which best described what his/her job is or was like (possible real status) in the context on a 7-point verbally anchored scale (that is, 7 alternatives); (b) the alternative which best described what the job should be or should have been like (want status) on the same 7-point verbally anchored scale; and (c) the degree of satisfaction of each of the seven alternatives, that is, the anticipated satisfaction (measured on a 21-point bipolar scale) if they engage in each alternative. From these ratings, item discrepancy was indicated by the gap between possible real alternative and want alternative, and was divided into five categories $(0$, $-1,-2,-3$, and -4 ; other discrepancy levels were ignored). In addition, the mean satisfaction score for the seven alternatives represented anticipated satisfaction that was used as the dependent variable. Their results showed that the relation between item discrepancy and item satisfaction was stronger for the higher important form (intrinsic) than that for lower important form (non-intrinsic) on three items. However, as in Study 2 of their research, many participants' data was eliminated prior to analysis, because their manipulation check reports did not correspond to the manipulation of question content involving intrinsic interest as more important. In addition, the limited range of discrepancy $(0,-1,-2,-3$, and -4) also eliminated many participants' data.

Finally, in Study 4 of Mobley and Locke's (1970) research, they manipulated the high-low importance by real situation, in which two quizzes were taken in class, one was the regular quiz (high importance condition), which was a real test for the class, and the other one was the research quiz (low importance condition), which was a research purpose test (grades would not be reported to the instructor). All participants took part in these two tests, and were asked to indicate their acceptable lowest grade on the test and the anticipated satisfaction if they get different level of grades. The gap of these two grades was used to index the level of have-want discrepancy. Finally, after knowing their actual test score, they were asked to rate their actual satisfaction for each test. As the previous study, Mobley and Locke (1970) compared the relation between discrepancy and actual satisfaction for high importance condition (regular quiz) and low importance condition (research 
quiz). The results also revealed that the relation between discrepancy and actual satisfaction for regular quiz was stronger than that for the research quiz. However, the result was only determined for participants with large discrepancy, not for the entire sample.

Therefore, although Locke (1969) and Mobley and Locke's (1970) withinsubject experiments supported the range-of-affect hypothesis, given the limitations of importance manipulation and data analysis, the conclusions should be viewed with caution. A more desirable experimental design was needed to examine Locke's hypothesis. Consequently, the purpose of this study was to examine the range-of-affect hypothesis by using a different experimental design. In the current study, level of importance was not indirectly manipulated by related factors, such as intrinsic interest or type of quiz in Mobley and Locke's (1970) study, but was mentioned directly in the scenario. This design approach prevented participants having different importance perception on manipulation. In addition, the discrepancy level in the current experiments was anchored by the gap between the ideal standard and possible real objects or status that is mentioned in the scenario. This design approach avoided the drawback resulting from participants' rating real and want status. These two design changes avoided the complex experiment procedure and data analysis used in Mobley and Locke's (1970) study.

Generally, the purpose of this study was to examine Locke's hypothesis in four different scenarios. In each scenario, two people were described as having the same want circumstance or state on the same objects defined by two dimensions. For example, in the first scenario, both $\mathrm{A}$ and $\mathrm{B}$ wanted "a large house in a convenient location", which was the want object defined by the two dimensions of size and location. However, in the scenario, $\mathrm{A}$ and $\mathrm{B}$ were described as having different opinions about the two dimensions. For example, A regarded size to be higher importance than location and B regarded location to be higher importance than size. After reading this information, participants were asked to rate the level of satisfaction on several proposed objects from the viewpoints of A and $\mathrm{B}$, respectively. The proposed objects were defined by different levels of the same two dimensions. For example, in the first scenario, the proposed houses included (a) a 30-ping ( 1 ping $\left.=3.3 \mathrm{~m}^{2}\right)$ house located in the city centre, (b) a 50-ping house located in the city centre, (c) a 30-ping house located in the suburbs, and (d) a 50-ping house located in the suburbs. These proposed houses were designed to anchor the level of discrepancy between have and want circumstances. For example, 30-ping and 50-ping houses were used to anchor the level of have-want 
discrepancy in the size dimension; a 50-ping house has a smaller discrepancy than a 30-ping house. City center and suburbs were used to anchor the level of have-want discrepancy in the location dimension; a house in the city centre has smaller discrepancy than a house in the suburbs (according to the need of convenient location). In order to avoid participants becoming aware of the manipulated level, several filter objects described with other dimensions were also included, such as "a 27-ping house with house management service, which is located in the city center and near good schools". Thus, in the scenario, dimension importance was manipulated by the viewpoint of $\mathrm{A}$ and $\mathrm{B}$, and have-want discrepancy of each dimension was anchored by the gap between proposed objects and the want circumstance. Finally, the dependent variable was measured as the satisfaction of each proposed object.

The main purpose of these experiments was to show if the relation between have-want discrepancy and proposed object satisfaction was stronger on the more important dimension than the less important dimension, depending on which viewpoint was taken. Specifically, in the first scenario, it was expected that, given the condition of house location, the relation between house size (30 and 50-ping) and object satisfaction would be stronger when participants took the viewpoint of $\mathrm{A}$ than those that took the viewpoint of B, since A placed more concern on the dimension of size. On the other hand, given the condition of size, it was expected that the relation between house location (city center and suburb) and object satisfaction would be stronger when participants took the viewpoint of $\mathrm{B}$ compared to those who took the viewpoint of A, since B was more concerned with the dimension of house location. In other words, it postulated a two-way interaction of viewpoint and have-want discrepancy for each dimension in predicting the object satisfaction in a $2 \times 2 \times 2$ (two viewpoints $\times$ high/low discrepancy on dimension $1 \times$ high/low discrepancy on dimension 2) within-subject ANOVA.

To sum up, the purpose of this study was to examine Locke's rangeof-affect hypothesis $(1969,1976)$ by a within-subject design to show if the association between have-want discrepancy and proposed object satisfaction was stronger on the more important dimension than the less important dimension for a person. If the hypothesis was supported, it could be concluded that satisfaction evaluation has incorporated the information of importance, and the basic assumption behind the importance-weighting procedure that importance judgment and satisfaction evaluation are two independent processes is not supported, implying that weighting satisfaction scores with importance scores is unnecessary. 


\section{METHOD}

\subsection{Participants and Procedure}

Forty undergraduate students $(27.5 \%$ male $[n=11]$ and $72.5 \%$ female $[n=29])$ at National Taiwan University participated in the study in exchange for an additional credit in a general psychology course. The mean age was 20.36 years $(\mathrm{SD}=2.04)$. The same questionnaire with two forms (described in the following section) was randomly assigned to the participants. Twenty participants completed each form. The questionnaire was filled out in approximately $30 \mathrm{~min}$ and then handed in to the researchers. Finally, researchers asked participants what they thought about the experiment to see if participants realized the manipulation and therefore deliberately gave artificial responses. No participants guessed the main purpose of the study.

\subsection{Instruments}

2.2.1. Scenario Questionnaires. In order to certify the generality of the results for various life areas, four scenarios were generated containing different life contents. The first scenario tapped living condition with house size and location. The second scenario involved health with physical condition and thinking. The third scenario referred to ability with academic and extracurricular achievement. Finally, the fourth scenario involved gaining supports from other-help and self-help when confronting problems.

In each scenario, two individuals with the same content of their want circumstance defined by two dimensions were described as having different importance perception on the two dimensions. After reading this information for each scenario, participants were asked to rate the degree of satisfaction of several objects on a 7-point Likert-type scale ranging from 1 (very dissatisfied) to 7 (very satisfied) from the viewpoint of each person portrayed in the scenario. These objects not only included the manipulated objects but also contained filter objects to avoid participants becoming aware of the researchers' purpose and to make the scenario authentic. In addition, the manipulated objects and filter objects were randomly arranged. The four scenarios were displayed in the same order for all participants because it is assumed that contents of the four scenarios do not interact with each other. But the order of viewpoint taken in each scenario was counterbalanced. In other words, in both forms, the four scenarios were displayed in the same order, but in one form, the viewpoint of A was taken first, and in the other form, the viewpoint of B was taken first (A and B represent the two individuals portrayed in each scenario). 
The content of the first scenario was described in the introduction, we did not repeated here. The content of the second scenario was that there are two old people who want to participate in an activity that could improve their physical condition and enhance their thinking ability (anchoring the want content). However, one of them regards physical condition to be more important than thinking ability and the other one regards thinking ability to be more important than physical condition (manipulating the importance perception). Participants were then asked to rate the degree of satisfaction on the subsequent list of activities from the perspective of the two people portrayed in the scenario, respectively. In these proposed activities, maintaining and improving physical condition were used to manipulate the high and low discrepancy on the body function dimension. Basic and advanced thinking ability were used to manipulate the high and low discrepancy on the thinking ability dimension. In addition to manipulated activities, several filter projects were also included.

The content of the third scenario was that there are two National Taiwan University students who want to have distinctive extracurricular achievement and good grades academically (anchoring the want content). However, one of them regards extracurricular achievement to be more important than academic achievement, the other one regards academic achievement to be more important than extracurricular achievement (manipulating the importance perception). In Taiwan, participating in clubs or not is usually a traded-off between extracurricular achievement and good grades for students. Therefore, the content of the third scenario has its merit and external validity in the current study. Participants were then asked to rate the degree of satisfaction on the following conditions from the perspective of the two people portrayed in the scenario, respectively. In these conditions, joining clubs and having distinctive achievement were used to manipulate the high and low discrepancy on the extracurricular achievement dimension. Bad and good grades were used to manipulate the high and low discrepancy on the academic achievement dimension. In addition to manipulated conditions, several filter projects were also included.

Finally, the content of the forth scenario was that there are two people who not only would like to get other-help but also want to have enough selfability when confronting problems (anchoring the want content). However, one of them regards other-help to be more important than self-help, the other one regards self-help to be more important than other-help (manipulating the importance perception). Participants were then asked to rate the degree of satisfaction on the subsequent situations from the perspective of the two people portrayed in the scenario, respectively. In these conditions, 
no other-help and gaining other-help were used to manipulate the high and low discrepancy on the others' help dimension. Without and with enough ability of self-help were used to manipulate the high and low discrepancy on the self-help dimension. In addition to manipulated situations, several filter projects were also included.

\section{RESULTS}

A $2 \times 2 \times 2$ (two viewpoints $\times$ high $/$ low discrepancy on dimension $1 \times \mathrm{high} /$ low discrepancy on dimension 2) within-subject ANOVA was conducted for each scenario. Because the main focus of the current study was to examine if the range-of-affect hypothesis would be supported, the core concern in the following analysis was to see if the two-way interactions of viewpoint and high/low discrepancy of two dimensions (namely, viewpoint $\times$ high/low discrepancy on dimension 1 and viewpoint $\times$ high/low discrepancy on dimension 2) were significant in the within-subject ANOVA model. Although other significant effects may represent its particular meaning in each scenario, because these effects have nothing to do with the examination and interpretation on the range-of-affect hypothesis, other significant effects were not interpreted in the following analysis for each scenario. However, a summary ANOVA table was provided for each scenario to display the whole effect in each within-subject ANOVA model.

For the first scenario ( $n=38$, two participants had a missing value in this scenario) the factors of viewpoint, house size and house location were regarded as fixed effects and subject factor was regarded as random effects. The within-subjects ANOVA summary table was provided in Table I, which revealed that there was a significant three-way interaction of viewpoint, house size, and house location.

Under the three-way interaction effect, further analysis was conducted to examine the simple two-way interaction effect between viewpoint and house size, and the simple two-way interaction effect between viewpoint and house location. The results revealed that, given the 30-ping house and 50-ping house, viewpoint and house location had significant interaction effects, $F(1,74)=17.22, \operatorname{MSE}=0.61, p<0.01$, partial $\eta^{2}=0.28$ and $F(1,74)=$ 52.73 , MSE $=0.61, p<0.01$, partial $\eta^{2}=0.54$, respectively. In addition, given the house location with the city center and suburbs, viewpoint and house size had significant interaction effects, $F(1,74)=89.47$, MSE $=0.52$, $p<0.01$, partial $\eta^{2}=0.70$ and $F(1,74)=36.98, \operatorname{MSE}=0.52, p<0.01$, partial $\eta^{2}=0.49$, respectively. 
TABLE I

ANOVA summary table for scenario 1

\begin{tabular}{lrrrll}
\hline Source & SS & df & MS & $F$ & Partial $\eta^{2}$ \\
\hline Subject & 43.61 & 37 & 1.18 & & \\
Viewpoint & 9.59 & 1 & 9.59 & $11.04^{* *}$ & 0.23 \\
Error & 32.16 & 37 & 0.87 & & \\
House size & 156.33 & 1 & 156.33 & $190.14^{* *}$ & 0.84 \\
Error & 30.42 & 37 & 0.82 & & \\
House location & 168.01 & 1 & 168.01 & $127.55^{* *}$ & 0.78 \\
Error & 48.74 & 37 & 1.32 & & \\
Viewpoint $\times$ House size & 62.65 & 1 & 62.65 & $107.28^{* *}$ & 0.74 \\
Error & 21.61 & 37 & 0.58 & & \\
Viewpoint $\times$ House location & 39.80 & 1 & 39.80 & $51.77^{* *}$ & 0.58 \\
Error & 28.45 & 37 & 0.77 & & \\
House size $\times$ House location & 1.59 & 1 & 1.59 & 3.54 & 0.09 \\
Error & 16.66 & 37 & 0.45 & & \\
Viewpoint $\times$ House size $\times$ House location & 2.96 & 1 & 2.96 & $6.52^{*}$ & 0.15 \\
Error & 16.79 & 37 & 0.45 & & \\
Total & 679.37 & 303 & & & \\
\hline
\end{tabular}

Note: ${ }^{*} p<0.05 .{ }^{* *} p<0.01$.

The interaction plots were presented in Figures 1a, b, 2a, and b. Figure 1a and $b$ showed that, given the level of house location, the slope of the viewpoint of house size was steeper than the slope of the viewpoint of house location, indicating that the degree of satisfaction was sensitive to the havewant discrepancy on the house size dimension when participants regarded house size to be more important. On the other hand, Figure $2 \mathrm{a}$ and $\mathrm{b}$ showed that given the level of house size, the degree of satisfaction was sensitive to the have-want discrepancy on the house location dimension when participants regarded the house location to be more important. Thus, both of the interaction patterns supported Locke's range-of-affect hypothesis $(1969,1976)$ that the relationship between have-want discrepancy and house satisfaction was stronger on more important dimensions than less important dimensions.

For the second scenario $(n=39)$, a $2 \times 2 \times 2$ within-subject ANOVA model was also used in which the factors of viewpoint, body function and thinking ability were regarded as fixed effects and subject factor was regarded as random effects. The within-subjects ANOVA summary table of this model was provided in Table II. It revealed the significant twoway interaction of viewpoints and body function, $F(1,38)=26.44$, 
(a) Houses at suburb

Satisfaction

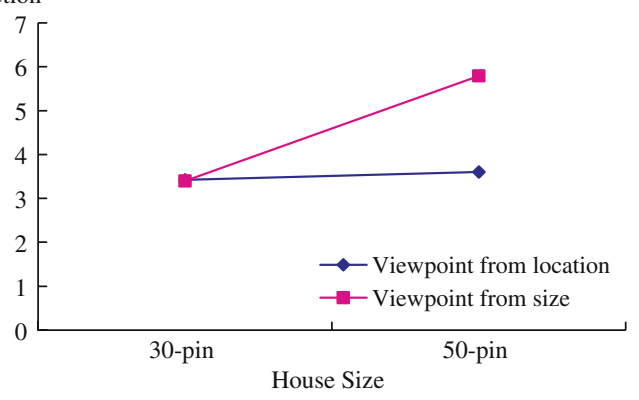

(b)

Houses at city centre

Satisfaction

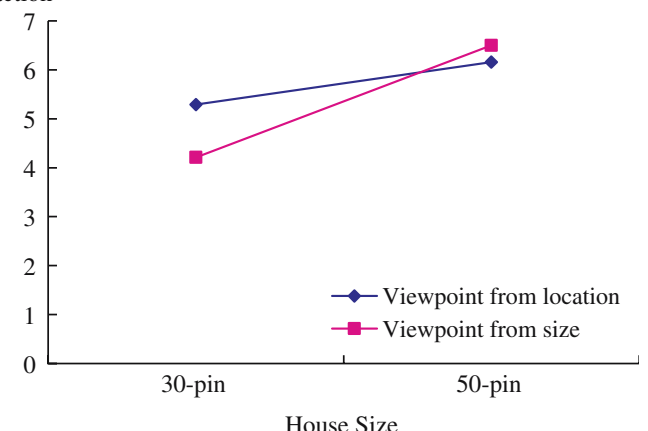

House Size

Figure 1. The interaction plot of viewpoint and house size given the level of houses at (a) suburb (b) City Centre.

MSE $=0.63, p<0.01$, partial $\eta^{2}=0.41$; and the significant two-way interaction of viewpoints and thinking ability, $F(1,38)=15.51$, MSE $=$ $0.79, p<0.01$, partial $\eta^{2}=0.29$, without the significant three-way interaction.

The interaction plots were presented in Figures 3 and 4. Figure 3 showed that the degree of satisfaction was sensitive to the have-want discrepancy of the body function dimension when participants regarded the body function to be more important. In Figure 4, the slope of the viewpoint of thinking ability was steeper than the slope of the viewpoint of body function, indicating that the degree of satisfaction was sensitive to the have-want discrepancy of thinking ability dimension when participants regarded thinking ability to be more important. This finding supported Locke's range-of-affect hypothesis $(1969,1976)$ as well.

The third scenario was a $2 \times 2 \times 2$ within-subject ANOVA design $(n=39)$, in which the factors of viewpoint, extracurricular achievement 
(a)

30-pin Houses

Satisfaction

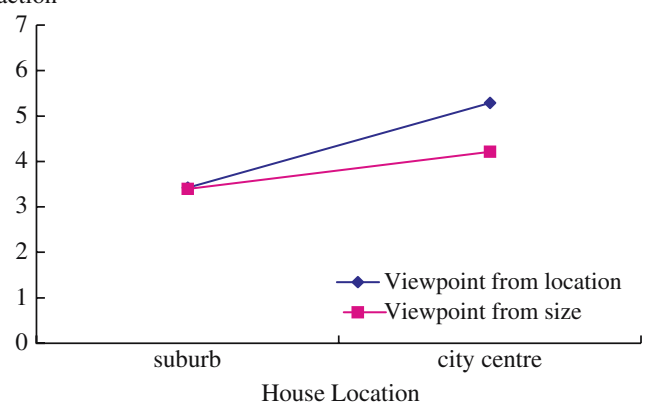

(b)

50-pin Houses

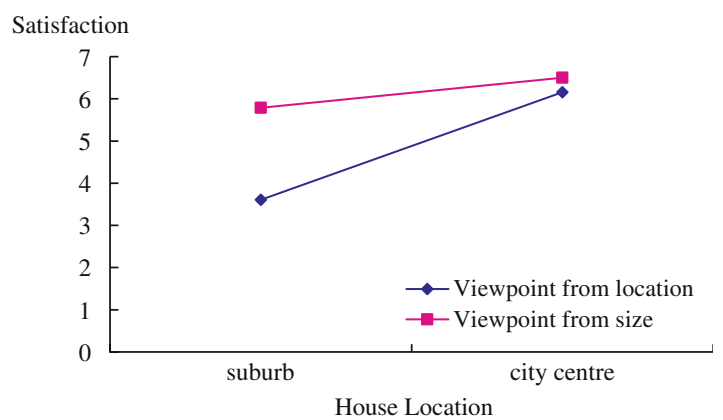

Figure 2. The interaction plot of viewpoint and house location given the level of a 30pin houses b 50pin houses.

and academic achievement, were regarded as fixed effects and subject factor was regarded as random effects. The within-subjects ANOVA summary table of this model was provided in Table III, which showed that the interaction of viewpoint and extracurricular achievement, $F(1,38)=18.03$, MSE $=0.46, p<0.01$, partial $\eta^{2}=0.51$; and the interaction of viewpoint and academic achievement were significant, $F(1,38)=102.70$, MSE $=$ $0.52, p<0.01$, partial $\eta^{2}=0.84$.

The interaction plots were presented in Figures 5 and 6 . Figure 5 showed that the slope of the viewpoint of extracurricular achievement was steeper than the slope of the viewpoint of academic achievement when participants regarded the extracurricular achievement to be more important. On the other hand, in Figure 6, the slope of the viewpoint of academic achievement was steeper than the slope of the viewpoint of extracurricular achievement when participants regarded the academic achievement to be more important. This finding also supported Locke's range-of-affect hypothesis $(1969,1976)$. 
TABLE II

ANOVA summary table for scenario 2

\begin{tabular}{lrrrll}
\hline Source & SS & df & MS & $F$ & Partial $\eta^{2}$ \\
\hline Subject & 126.88 & 38 & 3.34 & & \\
Viewpoint & 1.28 & 1 & 1.28 & 1.15 & 0.03 \\
Error & 42.22 & 38 & 1.11 & & \\
Body function & 4.15 & 1 & 4.15 & $5.99^{*}$ & 0.14 \\
Error & 26.35 & 38 & 0.69 & & \\
Thinking ability & 23.71 & 1 & 23.71 & $27.47^{* *}$ & 0.42 \\
Error & 32.80 & 38 & 0.86 & & \\
Viewpoint $\times$ Body function & 16.61 & 1 & 16.61 & $26.44^{* *}$ & 0.41 \\
Error & 23.89 & 38 & 0.63 & & \\
Viewpoint $\times$ Thinking ability & 12.32 & 1 & 12.32 & $15.51^{* *}$ & 0.29 \\
Error & 30.18 & 38 & 0.79 & & \\
Thinking ability $\times$ Body function & 0.12 & 1 & 0.12 & 0.29 & 0.01 \\
Error & 15.39 & 38 & 0.41 & & \\
Viewpoint $\times$ Thinking ability $\times$ Body function & 0.12 & 1 & 0.12 & 0.33 & 0.01 \\
Error & 13.39 & 38 & 0.35 & & \\
Total & 369.41 & 311 & & & \\
\hline
\end{tabular}

Note: ${ }^{*} p<0.05 .{ }^{* *} p<0.01$.

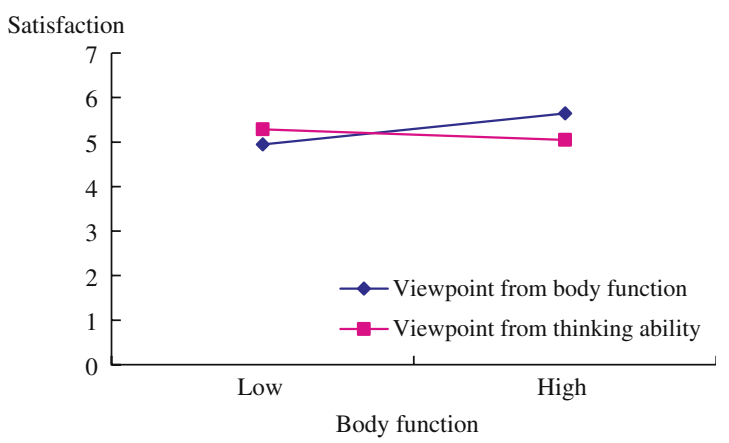

Figure 3. The interaction of viewpoint and body function.

Finally, in the fourth scenario $(n=39)$ the factors of viewpoint, otherhelp and self-help were regarded as fixed effects and subject factor was regarded as random effects. The $2 \times 2 \times 2$ within-subjects ANOVA summary table of this model was provided in Table IV, which revealed that the interaction of viewpoints and other-help, $F(1,38)=66.46, \mathrm{MSE}=0.57$, $p<0.01$, partial $\eta^{2}=0.75$; and the interaction of viewpoints and self-help were significant, $F(1,38)=32.05, \mathrm{MSE}=0.52, p<0.01$, partial $\eta^{2}=0.62$. 


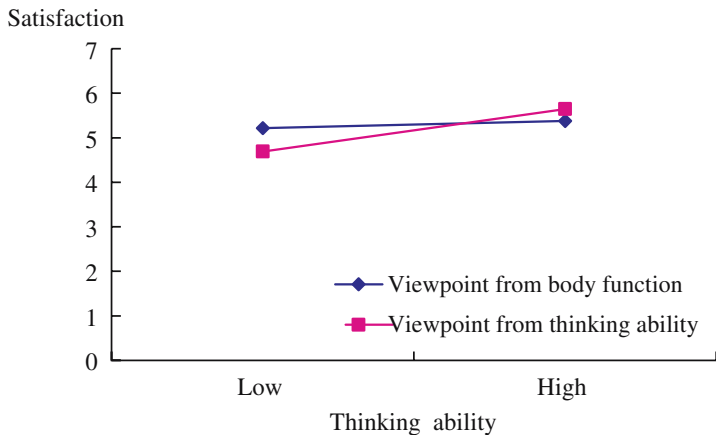

Figure 4. The interaction plot of viewpoint and thinking ability.

TABLE III

ANOVA summary table for scenario 3

\begin{tabular}{lrrrll}
\hline Source & SS & df & MS & \multicolumn{1}{l}{ P } & Partial $\eta^{2}$ \\
\hline Subjects & 31.72 & 38 & 0.83 & 1.35 & \\
Viewpoint & 23.16 & 1 & 23.16 & $42.99^{* *}$ & 0.53 \\
Error & 20.47 & 38 & 0.54 & & \\
Extracurricular achievement & 85.16 & 1 & 85.16 & $92.54^{* *}$ & 0.71 \\
Error & 34.97 & 38 & 0.92 & & \\
Academic achievement & 705.00 & 1 & 705.00 & $860.82^{* *}$ & 0.96 \\
Error & 31.12 & 38 & 0.82 & & \\
Viewpoint $\times$ Extracurricular achievement & 18.03 & 1 & 18.03 & $38.93 * *$ & 0.51 \\
Error & 17.60 & 38 & 0.46 & & \\
Viewpoint $\times$ Academic achievement & 102.70 & 1 & 102.70 & $195.81^{* *}$ & 0.84 \\
Error & 19.93 & 38 & 0.52 & & \\
Academic $\times$ Extracurricular achievement & 7.08 & 1 & 7.08 & $14.13 * *$ & 0.27 \\
Error & 19.05 & 38 & 0.50 & & \\
Viewpoint $\times$ Academic $\times$ Extracurricular & 0.72 & 1 & 0.72 & 1.31 & 0.03 \\
Error & 20.90 & 38 & 0.55 & & \\
Total & 1137.61 & 311 & & & \\
\hline
\end{tabular}

Note: ${ }^{*} p<0.05 .{ }^{* *} p<0.01$.

The interaction plots were presented in Figures 7 and 8. Figure 7 showed that the slope of the viewpoint of other-help was steeper than the slope of the viewpoint of self-help when participants regarded the other-help to be more important. On the other hand, in Figure 8 the slope of the viewpoint of self-help was steeper than the slope of the viewpoint of other-help when participants regarded the self-help to be more important. 


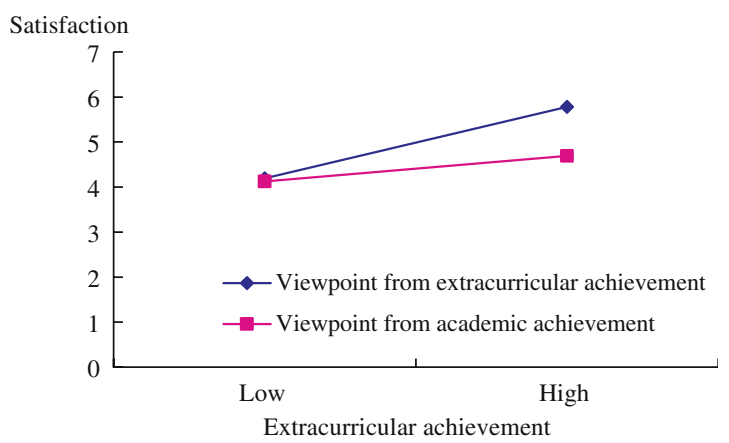

Figure 5. The interaction plot of viewpoint and extracurricular achievement.

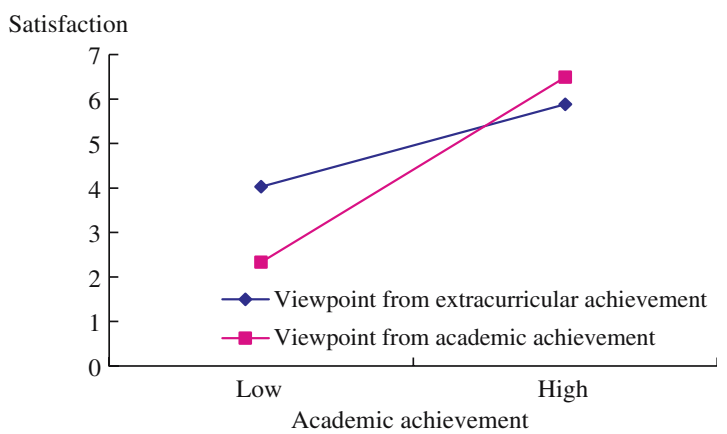

Figure 6. The interaction plot of viewpoint and academic achievement.

\section{DISCUSSION}

Importance weighting was a common procedure in assessing QOL. The basic idea of the procedure was that given a set of life areas, each area would have different importance to an individual's life, therefore, on assessing his/ her QOL, not only the current satisfaction of the life area should be evaluated, but the importance of that life area should also be considered. Accordingly, a QOL score was empirically defined as the importanceweighted satisfaction score of each item. This viewpoint implicitly implied that a satisfaction evaluation and an importance judgment are two independent processes and importance weighting would contribute to incorporating the meaning of importance into the overall life satisfaction score. However, according to Locke's range-of-affect hypothesis $(1969,1976)$ and previous empirical studies (Mobley and Locke, 1970; Rice et al., 1991a; Rice et al., 1991b; McFarlin and Rice, 1992; McFarlin et al., 1995; Wu and Yao, 
TABLE IV

ANOVA summary table for scenario 4

\begin{tabular}{lrrrll}
\hline Source & SS & df & MS & $F$ & Partial $\eta^{2}$ \\
\hline Subject & 49.10 & 38 & 1.29 & & \\
Viewpoint & 0.63 & 1 & 0.63 & 0.95 & 0.02 \\
Error & 25.12 & 38 & 0.66 & & \\
Other-help & 265.85 & 1 & 265.85 & $227.51^{* *}$ & 0.86 \\
Error & 44.40 & 38 & 1.17 & & \\
Self-help & 775.85 & 1 & 775.85 & $738.83^{* *}$ & 0.95 \\
Error & 39.90 & 38 & 1.05 & & \\
Viewpoint $\times$ Other-help & 66.46 & 1 & 66.46 & $115.91^{* *}$ & 0.75 \\
Error & 21.79 & 38 & 0.57 & & \\
Viewpoint $\times$ Self-help & 32.05 & 1 & 32.05 & $61.83^{* *}$ & 0.62 \\
Error & 19.70 & 38 & 0.52 & & \\
Self-help $\times$ Other-help & 1.04 & 1 & 1.04 & 0.91 & 0.02 \\
Error & 43.21 & 38 & 1.14 & & \\
Viewpoint $\times$ Self-help $\times$ Other-help & 0.01 & 1 & 0.01 & 0.02 & 0.00 \\
Error & 27.24 & 38 & 0.72 & & \\
Total & 1412.35 & 311 & & & \\
\hline
\end{tabular}

Note: ${ }^{*} p<0.05 .{ }^{* *} p<0.01$.

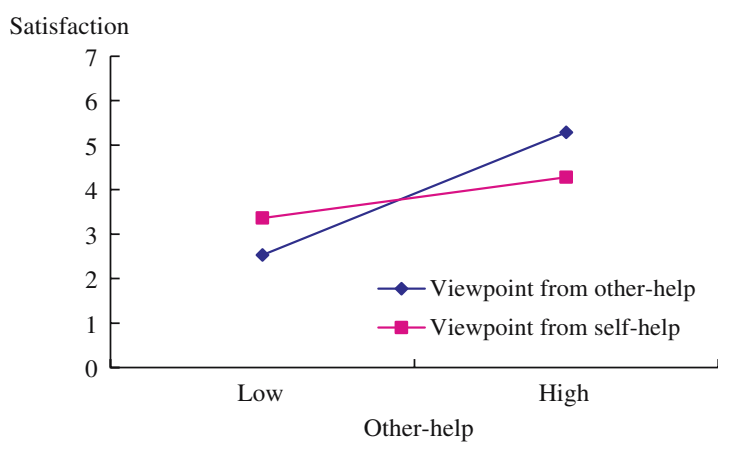

Figure 7. The interaction plot of viewpoint and other-help.

in press a), satisfaction rating has reflected the judgment of importance. Accordingly, the rationale for weighting satisfaction scores with importance scores may be unsound.

However, because previous studies examining Locke's range-of-affect hypothesis were conducted by between-subject designs and the within-subject experiments conducted by Locke (1969) and Mobley and Locke (1970) were not ideal designs for examining the hypothesis, the current study further examined Locke's hypothesis with a new within-subject design. Four 


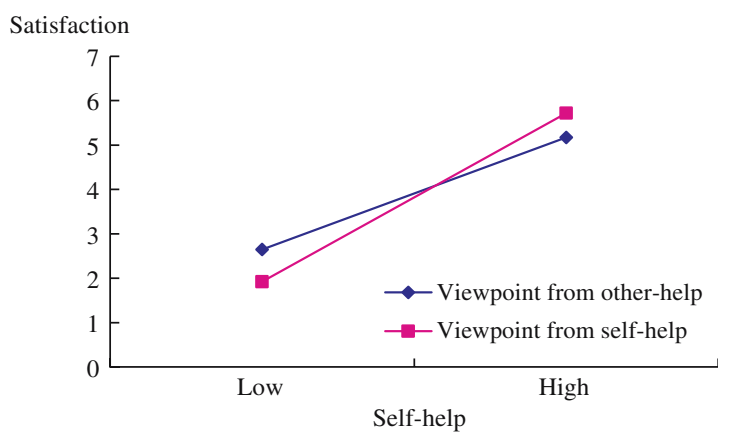

Figure 8. The interaction plot of viewpoint and self-help.

scenarios were designed to investigate if the association between have-want discrepancy and proposed object satisfaction would be stronger on the more important dimension than less important dimension. According to the results of the four scenarios, Locke's hypothesis was supported. In each scenario, the association between have-want discrepancy on a dimension and object satisfaction was stronger when participants viewed the dimension to be more important. In other words, when participants regard one dimension to be more important than the other dimension, they were sensitive to the gap between have and want circumstances of that dimension, and then, the degree of satisfaction was affected. This finding revealed that proposed object satisfaction reflects the effect of have-want discrepancy and the importance judgment of the dimension that it relies on. This finding implied that satisfaction evaluation incorporates the information of importance, and therefore, weighting satisfaction scores with importance scores is unnecessary. Actually, many empirical studies have indicated that importance-weighted satisfaction scores were not superior to unweighted satisfaction scores in predicting criterion variables, such as employee turnover (e.g., Mikes and Hulin, 1968; Waters and Roach, 1971), global job satisfaction (e.g., Ewen, 1967; Rice et al., 1991a; Staples and Higgins, 1998; Waters, 1969; Waters and Roach, 1971), and global life satisfaction (Hsieh, 2003; Wu and Yao, in press b), suggesting that weighting satisfaction scores with importance scores offers no benefit.

In addition to Locke's hypothesis, the results of this study also revealed that the interaction patterns were not the same for each scenario. The same finding was also observed in Rice et al. (1991) study, in which they concluded that different content might yield differences in the specific form of the discrepancy and importance interaction, even although the general form 
of the interaction is consistent with the range-of-affect hypothesis. In other words, in addition to the general interaction pattern of discrepancy and importance, each investigated facet or life areas would also bring their indigenous characteristics to forming the observed interaction pattern. Although this information may not be crucial in examining Locke's hypothesis, when considering specific facets it could play an important role in understanding participants' perception and feelings concerning those facets. For example, in QOL research, it may contribute to understanding how patients perceive pain relief and health care in evaluating their life satisfaction in their current condition. However, in the current study examining Locke's hypotheses, the specific meaning of each area was not of concern. Consequently, the discussion of specific form of each interaction was not provided.

Hitherto, the focus of this research has been on the moderating effect of importance between the have-want discrepancy and satisfaction evaluation. However, Baron and Kenny (1986) indicated that a moderating effect is descriptive. A moderating effect only describes a phenomenon; it does not explain a psychological mechanism. Therefore, an interesting issue beyond Locke's range-of-affect hypothesis $(1969,1976)$ is why importance has moderating effect on the relationship between have-want discrepancy and satisfaction. Further studies are needed to figure out the psychological mechanism underlying the phenomenon described in Locke's hypothesis.

Finally, a major limitation of this study is that the scenario experiments did not allow participants to give their importance judgment on the dimensions set in each scenario. Thus, results of this study only demonstrate that when a subject is told how hypothetical people feel about a particular issue (e.g., size vs. location of a house), that subject is able to evaluate the satisfaction from the perspective of those hypothetical people. This is a main drawback for adopting scenario experiments, in which participants lie in hypothetical situations. Although the current results cannot directly lead to a strong conclusion that participants have generated and considered the information of importance when they make a satisfaction evaluation, however, the current results do show that altering participants' importance perceptions of external materials can affect satisfaction evaluations on the materials, at least implying that importance judgment and satisfaction evaluation are not two independent processes that is taken as a basic assumption behind the importanceweighting procedure. In other words, results of the current study still have a contribution to the issue of importance weighting. However, in future studies, experiments that allow participants to rely on their importance judgments when making satisfaction evaluations are needed to draw a cogent conclusion. 


\section{ACKNOWLEDGEMENTS}

We thank Li-jen Weng, Luo Lu, Chien-chih Kuo, and Chung-ping Cheng for their comments on the original version of the Master's Thesis. We also thank Cheryl Sheridan for her help in editing the English. This study was supported by National Science Council (NSC 94-2413-H-002-018) and the National Health Research Institute (NHRI-EX94-9204PP).

\section{REFERENCES}

Baron, R. M. and D. A. Kenny: 1986, 'The moderator-mediator variable distinction in social psychological research: Conceptual, strategic and statistical considerations', Journal of Personality and Social Psychology 51, pp. 1173-1182.

Cummins, R. A.: 1997, Comprehensive Quality of Life Scale - Adult: Manual (Deakin University, Australia).

Dijkers, M. P.: 2003, 'Individualization in quality of life measurement: Instruments and approaches', Archives of Physical Medicine and Rehabilitation 84, pp. S3-14.

Ewen, R. B.: 1967, 'Weighting components of job satisfaction', Journal of Applied Psychology 51, pp. 68-73.

Ferrans, C. and M. Powers: 1985, 'Quality of life index: Development and psychometric properties', Advances in Nursing Science 8, pp. 15-24.

Frisch, M. B.: 1992, 'Use of the quality of life inventory in problem assessment and treatment planning for cognitive therapy of depression', in A. Freeman and F.M. Dattlio (eds.), Comprehensive Casebook of Cognitive Therapy (Plenum Press, New York), pp. 27-52.

Frisch, M. B., J. Cornell, M. Villanueva and P. J. Retzlaff: 1992, 'Clinical validation of the quality of life inventory: A measure of life satisfaction for use in treatment planning and outcome assessment', Psychological Assessment 4, pp. 92-101.

Hsieh, C. M.: 2003, 'Counting importance: The case of life satisfaction and relative domain importance', Social Indicators Research 61, pp. 227-240.

Locke, E. A.: 1969, 'What is job satisfaction?', Organizational Behavior and Human Performance 4, pp. 309-336.

Locke, E. A.: 1976, 'The nature and causes of job satisfaction', in M.D. Dunnette (ed.), Handbook of Industrial and Organizational Psychology (Rand McNally, Chicago), pp. 1297-1343.

McFarlin, D. B., E. A. Coster, R. W. Rice and T. Coopper-Alison: 1995, 'Facet importance and job satisfaction: Another look at the range of affect hypothesis', Basic and Applied Social Psychology 16, pp. 489-502.

McFarlin, D. B. and R. W. Rice: 1992, 'The role of facet importance as a moderator in job satisfaction processes', Journal of Organizational Behavior 13, pp. 41-54.

Mikes, P. S. and C. L. Hulin: 1968, 'Use of importance as weighting component of job satisfaction', Journal of Applied Psychology 52, pp. 394-398.

Mobley, W. H. and E. A. Locke: 1970, 'The relationship of value importance to satisfaction', Organisational Behavior and Human Performance 5, pp. 463-483.

Raphael, D., E. Rukholm, I. Brown, P. Hill-Bailey and E. Donato: 1996, 'The Quality Of Life Profile-Adolescent Version: Background, description, and initial validation', Journal of Adolescent Health 19, pp. 366-375.

Rice, R. W., D. A. Gentile and D. B. McFarlin: 1991a, 'Facet importance and job satisfaction', Journal of Applied Psychology 76, pp. 31-39. 
Rice, R. W., K. Markus, R. P. Moyer and D. B. McFarlin: 1991b, 'Facet importance and job satisfaction: Two experimental tests of Locke's range of affect hypothesis', Journal of Applied Social Psychology 21, pp. 1977-1987.

Staples, D. S. and C. A. Higgins: 1998, 'A study of the impact of factor importance weightings on job satisfaction measures', Journal of Business and Psychology 13, pp. 211-232.

Waters, L. K.: 1969, 'The utility of importance weights in predicting overall job satisfaction and dissatisfaction', Educational and Psychological Measurement 29, pp. 519-522.

Waters, L. K. and D. Roach: 1971, 'Comparison of unweighted and importance-weighted job satisfaction measures for three samples of female office workers', Psychological Reports 28, pp. $779-782$.

Wu, C. H. and Yao G. (in press a) Do we need to weight item satisfaction by item importance? A perspective from Locke's range-of-affect hypothesis. Social Indicators Research.

Wu, C. H. and Yao G. (in press b) Do we need to weight satisfaction scores with importance ratings in measuring quality of life? Social Indicators Research.

Department of Psychology

G. Yao

National Taiwan University

1, Sec. 4, Roosevelt RD.

Taipei, Taiwan

E-mail: kaiping@ntu.edu.tw 